\title{
Comportamento sedentário associado ao tempo de tela em acadêmicos de educação física
}

\author{
Associated behavior at time of screening in physical \\ education academics
}

\author{
Jéssica dos Santos Martins \\ Michele Gonçalves Romcy Torres \\ Rayane Alves de Oliveira.
}

\section{RESUMO}

Introdução: Atualmente o tempo de tela é importante indicador de atividades sedentárias. A explosão nos últimos anos de acesso à internet e o avanço da tecnologia vem contribuindo significativamente para o comportamento sedentário. A premissa é que quanto maior for o tempo de tela, menor será a prática de atividade física. Objetivo: Investigar o comportamento sedentário associado ao tempo de tela em acadêmicos de Educação Física. Métodos: Estudo de delineamento transversal e natureza quantitativa realizado nas Instituições de Ensino Superior reconhecidas pelo Ministério de Educação (MEC) e com sede própria na capital de Fortaleza e Região Metropolitana do Ceará. Para investigação do nível de atividade física e comportamento sedentário foi utilizado o Questionário Internacional de Atividade Física (IPAQ - versão curta) e para investigação do tempo de tela, questionário semiestruturado com perguntas relacionadas ao tempo despendido na utilização de televisão, videogame, computador (tablets; ipad e outros tipos de computador portátil) e celular. Resultados: Participaram do estudo 887 acadêmicos de Educação Física, sendo 600 (67,6\%) do sexo masculino. A maior parte da amostra foi de acadêmicos com idades entre 19 e 23 anos, do turno da noite e que trabalhavam. A prevalência de comportamento sedentário nos acadêmicos que apresentavam tempo sentado superior a $\geq 6$ horas semanais foi de $94,6 \%$ e sua associação foi positiva quando relacionado ao tempo de tela. Conclusões: $O$ comportamento sedentário esteve diretamente associado ao tempo de tela. Desta forma, estudos que associem diversos fatores e indicadores de tempo de tela tornam-se importantes para direcionamentos futuros de políticas públicas voltadas à saúde e combate ao comportamento sedentário.

\section{PALAVRAS-CHAVE:}

Comportamento sedentário; Tempo de tela; Acadêmicos.

${ }^{1}$ Faculdade Terra Nordeste - FATENE. 


\section{ABSTRACT}

Introduction: Currently, screen time is an important indicator of sedentary activities. The explosion in recent years of internet access and the advancement of technology has significantly contributing to sedentary behavior. The premise is that the greater the display time, the lower the physical activity. Objective: To investigate the behavior associated with the sedentary screen time in academic physical education. Methods: Cross-sectional study and quantitative carried out in higher education institutions recognized by the Ministry of Education (MEC) and its own headquarters in the city of Fortaleza-Ceara and Metropolitan region. To investigate the level of physical activity and sedentary behavior we used the International Physical Activity Questionnaire (short IPAQ-version) and for screen time of the research, semi-structured questionnaire with questions related to the time spent in the use of television, video game, computer (tablets, ipad and other laptop) and mobile. Results: The study included 887 students of Physical Education, and 600 (67.6\%) were male. Most of the sample consisted of academics aged 19 and 23, the night shift and working. The prevalence of sedentary behavior in academic who had higher sitting time to $\geq 6$ hours per week was $94.6 \%$ and its association was positive when related to screen time. Conclusions: Sedentary behavior showed a positive association with the screen time. Thus, studies involving various factors and screen time indicators become important for future directions of public policies for health and combat sedentary behavior.

\section{KEYWORDS:}

Sedentary behavior; Screen time; Academics. 


\section{INTRODUÇÃO}

O comportamento sedentário (CS) associado ao tempo de tela (TT) é um dos principais fatores de risco para a mortalidade, especialmente por doenças cardiovasculares (VEERMAN et al., 2012; WORLD HEALTH ORGANIZATION, 2010), estando relacionado à redução da qualidade de vida da população (FONTES; VIANNA, 2009). Atualmente, o tempo de tela (TT) é importante indicador de atividades sedentárias, sobretudo, porque o tempo destinado na realização de atividades físicas tem sido dedicado às telas.

Não obstante, o avanço da tecnologia e a explosão nos últimos anos de acesso à internet vêm contribuindo significativamente para o CS. A premissa é que quanto maior for o TT, menor será a prática de atividade física. Assim, a associação entre o baixo nível de atividade física e TT, são hoje considerados os principais problemas de saúde pública nos países industrializados (SCHMID; RICCI; LEITZMANN, 2015).

No Brasil, os estudos relacionados ao TT abrangem diferentes públicos, bem como os fatores associados, dentre os quais se podem citar: CS, prática de atividade física, hábitos alimentares e obesidade (VASCONCELLOS, 2013). Uma recente pesquisa realizada com 20.000 universitários do Sul do Brasil avaliou os fatores que estão relacionados à compra de tablets (computador portátil). O fator que apresentou maior índice foi o de representação social, ou seja, o consumidor transmite e comunica à sociedade e para as pessoas de convívio, sua identidade e personalidade por meio da utilização do produto. Desta forma, as barreiras que estão ligadas à tecnologia, perpassam o simples acesso ao produto e podem revelar o perfil de uma nova geração (MEDEIROS; VIEIRA; NOGAMI, 2013).

Assim, o mundo virtual vai, progressivamente, confundindo os seus limites. A internet, o telefone celular e muitos novos equipamentos de tecnologia da informação vão transformando os comportamentos e as formas de se relacionar, ao passo em que comprometem a saúde e qualidade de vida dos indivíduos.

Corroborando com esta afirmação, um estudo realizado no Nordeste do Brasil que associou o tempo de tela à prática de atividade física revelou que $28,2 \%$ da amostra despendia mais de duas horas em atividades sedentárias (TV e computador), ratificando uma redução nos níveis de atividade física e consequentemente elevando o CS (OLIVEIRA et al., 2010).

Todavia, embora o TT tenha sido alvo de investigação nos últimos anos pelo avanço da tecnologia, grande parte das pesquisas se limita a avaliar sua associação apenas entre crianças e adolescentes em idade escolar. Outrossim, os indicadores de TT (televisão, computador, vídeo game, celular) variam de acordo com o fator associado investigado. Considerando esses aspectos, o presente estudo teve como objetivo investigar o CS associado ao TT em acadêmicos de Educação Física.

\section{METODOLOGIA}

\section{Planejamento da Pesquisa}

Este estudo apresentou delineamento transversal e natureza quantitativa, realizado com acadêmicos do $1^{\circ}$ ao $8^{\circ}$ semestre de todos os turnos matriculados no curso de Educação Física nas Instituições de Ensino Superior (IES) da capital de Fortaleza e Região questionário para investigação do nível de atividade física/comportamento sedentário e outro questionário semiestruturado para verificar a associação com o tempo de tela, ambos em um único arquivo. A participação dos acadêmicos foi autorizada mediante assinatura do Termo de Consentimento Livre e Esclarecido (TCLE) e o acesso às IES foi consentido mediante assinatura do termo de autorização.

\section{Local do Estudo}

Nesse estudo foram selecionadas as Universidades e Faculdades de Fortaleza e Região Metropolitana do Ceará.

Critério de Inclusão:

- Reconhecida pelo Ministério da Educação (MEC);

- Oferta do curso de Educação Física (Bacharel e/ou Licenciatura);

- IES particulares e públicas.

- Critério de Exclusão:

- IES que não possuíam sede própria; 
- IES em greve;

- IES que não fecharam turma;

- Oferta do curso à distância (EAD).

No total, 09 IES estão localizadas na cidade de Fortaleza e 01 na Região Metropolitana, distribuídas conforme quadro abaixo:

Quadro 01. Relação de Instituições de Ensino Superior reconhecidas pelo MEC na cidade de Fortaleza e Região Metropolitana.

\begin{tabular}{|l|l|}
\hline FORTALEZA & $\begin{array}{l}\text { REGIÃO } \\
\text { METROPOLITANA }\end{array}$ \\
\hline UNIFOR & \\
FIC & \\
UFC & \\
UECE & \\
FGF & FATENE \\
FANOR & \\
FAECE & \\
FATE & \\
FAMETRO & \\
\hline
\end{tabular}

A escolha do locus foi motivada pela escassez de estudos abrangentes sobre comportamento sedentário com esta população, ratificando ser o primeiro estudo realizado com toda a população de acadêmicos de Educação Física da capital de Fortaleza e Região Metropolitana do Ceará. No total a amostra de acadêmicos foi constituída conforme tabela 1 :

Tabela 1. Recrutamento de acadêmicos em Fortaleza e Região Metropolitana.

\begin{tabular}{lll}
\hline Variáveis & $\begin{array}{l}\text { Total } \\
\end{array}$ & $\mathrm{N}$ \\
\hline Instituição & & \\
FANOR & 39 & 4,4 \\
FAMETRO & 103 & 11,6 \\
FGF & 163 & 18,4 \\
FATENE & 248 & 28,0 \\
FIC & 196 & 22,1 \\
UNIFOR & 11 & 1,2 \\
UECE & 127 & 14,3 \\
Tipo & & \\
Particular & 760 & 85,7 \\
Pública & 127 & 14,3 \\
\hline
\end{tabular}

\section{Delineamento Amostral}

Devido à inacessibilidade a toda população, uma vez que alguns acadêmicos se encontravam em atividades nas quais não era possível a realização da coleta dos dados, os integrantes foram escolhidos de maneira aleatória simples, utilizando-se para tanto a lista de frequência.

Assim, a população desse estudo foi composta por 887 acadêmicos com a média de idade entre 18 e 34 anos, sendo 600 do sexo masculino e 227 do sexo feminino, matriculados no Ensino Superior no curso de Educação Física da capital de Fortaleza e Região Metropolitana do Ceará.

\section{INSTRUMENTOS E PROCEDIMENTOS}

Para investigação do nível de atividade física e comportamento sedentário foi utilizado o Questionário Internacional de Atividade Física (IPAQ - versão curta 2005). O IPAQ é um questionário que avalia o nível de atividade física numa semana típica, por meio da frequência e duração em minutos. Composto por três questões relacionadas aos tipos de atividade física e duas relacionadas ao comportamento sedentário, o questionário divide a prática em duas intensidades distintas (vigorosas e moderadas), a saber: i) vigorosas: são as atividades que precisam de um grande esforço físico e fazem respirar muito mais forte que o normal; ii) moderadas: são as atividades que precisam de algum esforço físico e fazem respirar um pouco mais forte que o normal. O protocolo de classificação do nível de atividade física foi realizado de acordo com a orientação do próprio IPAQ, que divide e conceitua as categorias em:

Sedentário - Não realiza nenhuma atividade física por pelo menos 10 minutos contínuos durante a semana;

Insuficientemente Ativo - Consiste em classificar os indivíduos que praticam atividades físicas por pelo menos 10 minutos contínuos por semana, porém de maneira insuficiente para ser classificado como ativos. Para classificar os indivíduos nesse critério, são somadas a duração e a frequência dos diferentes tipos de atividades (caminhadas + moderada + vigorosa). Essa categoria divide-se em dois grupos: 
- Insuficientemente Ativo A - Realiza 10 minutos contínuos de atividade física, seguindo pelo menos um dos critérios citados: frequência - 5 dias/semana ou duração - 150 minutos/semana;

- Insuficientemente Ativo B - Não atinge nenhum dos critérios da recomendação citada nos indivíduos insuficientemente ativos $A$;

Ativo - Cumpre as seguintes recomendações: a) atividade física vigorosa $-\geq 3$ dias/semana e $\geq 20$ minutos/sessão; b) moderada ou caminhada $-\geq 5$ dias/semana e $\geq 30$ minutos/sessão; c) qualquer atividade somada: $\geq 5$ dias/semana e $\geq 150 \mathrm{~min} / \mathrm{semana}$;

Muito Ativo - Cumpre as seguintes recomendações: a) vigorosa- $\geq 5$ dias/semana e $\geq 30 \mathrm{~min} / \mathrm{ses}-$ são; b) vigorosa $-\geq 3$ dias/semana e $\geq 20 \mathrm{~min} /$ sessão + moderada e ou caminhada $\geq 5$ dias/semana e $\geq$ $30 \mathrm{~min} /$ sessão.

O ponto de corte para comportamento sedentário foi o tempo sentado por semana $\geq 6$ horas/semanais (ARMSTRONG; BAUMAN; DAVIES, 2000). Para investigação do tempo de tela, foi aplicado um questionário semiestruturado com perguntas relacionadas ao tempo despendido na utilização de TV, VG, celular e computador (tablets; ipad e outros tipos de computador portátil), elaborado com base no IPAQ.

Além disto, a pesquisa abrangeu as seguintes etapas: i) Assinatura do termo de autorização para pesquisa e acesso ao campus (IES); ii) Assinatura do TCLE (acadêmicos).

\section{Análise Estatística}

Inicialmente, visando verificar se existia um relacionamento entre determinadas variáveis (sexo, idade, turno e status ocupacional) e o tempo em que os pesquisados dedicam à televisão, ao videogame, aos aplicativos do celular e ao computador, recorreu-se ao teste do qui-quadrado de Pearson, pois ele "detecta se existe uma associação significativa entre duas variáveis categóricas" (FIELD, 2009).

Em seguida, com vistas a constar o modo como o comportamento sedentário e o tempo de tela estão relacionados recorreu-se ao coeficiente de correlação de Spearman.

O cálculo do teste do qui-quadrado e do coeficiente de correlação de Spearman foram realizados com o auxílio do programa SPSS (Statistical Package for the Social Sciences) for Windows versão 20.0 que também auxiliou na análise descritiva. O nível de significância foi determinado em $5 \%(p<0,05)$. A duração diária tempo de tela (tempo de TV, celular, videogame ou computador) foi autoreportado. Sexo, idade, turno e condição de trabalho (status ocupacional) foram potenciais fatores associados ao CS.

\section{RESULTADOS}

Participaram do estudo 887 acadêmicos de Educação Física, sendo 600 (67,6\%) do sexo masculino. A maior parte da amostra foi de acadêmicos com idades entre 19 e 23 anos, do turno da noite e que trabalhavam. Os indivíduos em sua maioria foram classificados como ativos (Tabela 2).

Tabela 2. Características sociodemográficas e comportamentais de 887 acadêmicos de Educação Física participantes do estudo.

\begin{tabular}{|c|c|c|}
\hline \multirow[t]{2}{*}{ Variáveis } & \multicolumn{2}{|l|}{ Total } \\
\hline & $\mathrm{N}$ & $\%$ \\
\hline \multicolumn{3}{|l|}{ Sexo } \\
\hline Masculino & 600 & \\
\hline Feminino & 287 & 32,4 \\
\hline \multicolumn{3}{|l|}{ Idade } \\
\hline Até 18 anos & 58 & J \\
\hline $19-23$ anos & 409 & 46,1 \\
\hline $24-28$ anos & 207 & 23,3 \\
\hline 29 - 33 anos & 124 & 14,0 \\
\hline$\geq 34$ anos & 89 & 10,0 \\
\hline \multicolumn{3}{|l|}{ Tuno } \\
\hline Manhã & 322 & 36,3 \\
\hline Tarde & 116 & 13,1 \\
\hline Noite & 449 & \\
\hline \multicolumn{3}{|l|}{ Trabalho } \\
\hline Sim & 630 & \\
\hline Não & 257 & 29,0 \\
\hline \multicolumn{3}{|l|}{ NAF } \\
\hline Sedentário & 20 & 2,3 \\
\hline Insuficiente ativo $A$ & 79 & 8,9 \\
\hline Insuficiente ativo B & 74 & 8,3 \\
\hline Ativo & 387 & \\
\hline Muito ativo & 327 & 36,9 \\
\hline
\end{tabular}


O nível de atividade física não apresentou associação significativa quando comparado com sexo, idade, turno ou condição de trabalho e não esteve associado ao uso excessivo de telas.

A prevalência de CS nos acadêmicos que apresentavam tempo sentado superior $a \geq 6$ horas semanais foi de $94,6 \%$ e sua associação foi positiva quando relacionado ao TT. Embora o CS tenha apresentado significância com a idade, não foram identificadas interações entre as variáveis: sexo, turno e condição de trabalho (Tabela 3).

Tabela 3. Frequência absoluta e relativa do CS (tempo sentado) de acordo com as variáveis independentes.

\begin{tabular}{|c|c|c|c|c|c|}
\hline \multirow[t]{3}{*}{ Variáveis } & \multicolumn{4}{|c|}{$\begin{array}{l}\text { Comportamento } \\
\text { Sedentário }\end{array}$} & \multirow{3}{*}{$p^{*}$} \\
\hline & \multicolumn{2}{|c|}{$\begin{array}{l}<6 \text { horas/ } \\
\text { semana }\end{array}$} & \multicolumn{2}{|c|}{$\begin{array}{l}\geq 6 \text { horas/ } \\
\text { semana }\end{array}$} & \\
\hline & $\mathrm{n}$ & $\%$ & $\mathrm{~N}$ & $\%$ & \\
\hline \multicolumn{6}{|l|}{ Sexo } \\
\hline Masculino & 29 & 60,4 & 571 & 68,1 & \multirow{2}{*}{0,27} \\
\hline Feminino & 19 & 39,6 & 268 & 31,9 & \\
\hline
\end{tabular}

$\begin{array}{lllll}\text { Até 18 anos } & 3 & 6,2 & 55 & 6,6 \\ 19-23 \text { anos } & 17 & 35,4 & 392 & 46,7 \\ 24-28 \text { anos } & 16 & 33,3 & 191 & 22,8 \\ 29-33 \text { anos } & 1 & 2,1 & 123 & 14,7 \\ \geq 34 \text { anos } & 11 & 22,9 & 78 & 9,3\end{array}$

Turno

\begin{tabular}{llllll} 
Manhã & 17 & 35,4 & 305 & 36,4 & \\
Tarde & 7 & 14,6 & 109 & 13,0 & 0,95 \\
Noite & 24 & 50,0 & 425 & 50,7 & \\
Trabalho & & & & & \\
Sim & 36 & 75,0 & 594 & 70,8 & \\
Não & 12 & 25,0 & 245 & 29,2 & 0,53 \\
Tempo & 48 & 5,4 & 839 & 94,6 & 0,00 \\
sentado & & & & & \\
\hline
\end{tabular}

O tempo mediano de TT foi de 937,57 $\pm 2423,99$ $\mathrm{min} /$ totais e a forma de entretenimento mais utilizada foi o celular. Em linhas gerais, o uso do celular esteve associado às variáveis idade e condição de trabalho; onde $67,2 \%$ dos indivíduos com até 18 anos de idade utilizavam o celular por mais de 3 horas/dia e $65 \%$ não trabalhavam (vide tabela 4).

\section{DISCUSSÃO}

Este estudo investigou o comportamento sedentário associado ao tempo de tela em acadêmicos de Educação Física de Fortaleza e Região Metropolitana do Ceará. De modo geral, fatores sociodemográficos e comportamentais (idade, comportamento sedentário), estiveram associados ao TT entre os acadêmicos.

O nível de atividade física não esteve relacionado ao TT, o que pode ser justificado pelo fato da amostra ser composta por acadêmicos do curso de Educação Física. Visto que de modo geral o maior percentual foi classificado como indivíduos ativos. Esse fato chama bastante atenção, porque, embora a prática de atividade física esteja presente entre esta população, estudos similares realizados com acadêmicos afins da área da saúde, apresentaram um baixo nível de atividade física, revelando que suas preferências nem sempre estão ligadas à área que atuam (JÚNIOR et al., 2012; SILVA et al., 2007; FONTES; VIANNA, 2009).

Isto ocorre porque quando se fala em relacionamento entre duas variáveis sabemos que elas podem estar relacionadas de três formas, a saber: 1) podem estar positivamente relacionadas; 2) podem não estar relacionadas, o que equivale a dizer que uma é independente da outra; 3) podem estar negativamente relacionadas (FIELD, 2009).

Não obstante, a diferença encontrada em outros cursos pode ser atribuída ao fato de os acadêmicos de Educação Física, praticarem atividade física como parte da própria grade curricular, o que pode ter superestimado os valores descritos por eles (STAINO et al., 2010).

Diferença entre os sexos quanto aos níveis de atividade física não foram detectadas, diferente do estudo realizado com universitários em Curitiba, que detectou maior nível de atividade física entre os homens (CIESLACK et al., 2012). Outrossim, a idade também não esteve associada ao nível de atividade física. Exceção constitui nos critérios adotados para o CS, visto que a idade apresentou associação positiva, corroborando com o estudo realizado em Cuiabá (DIAS et al., 2014). 


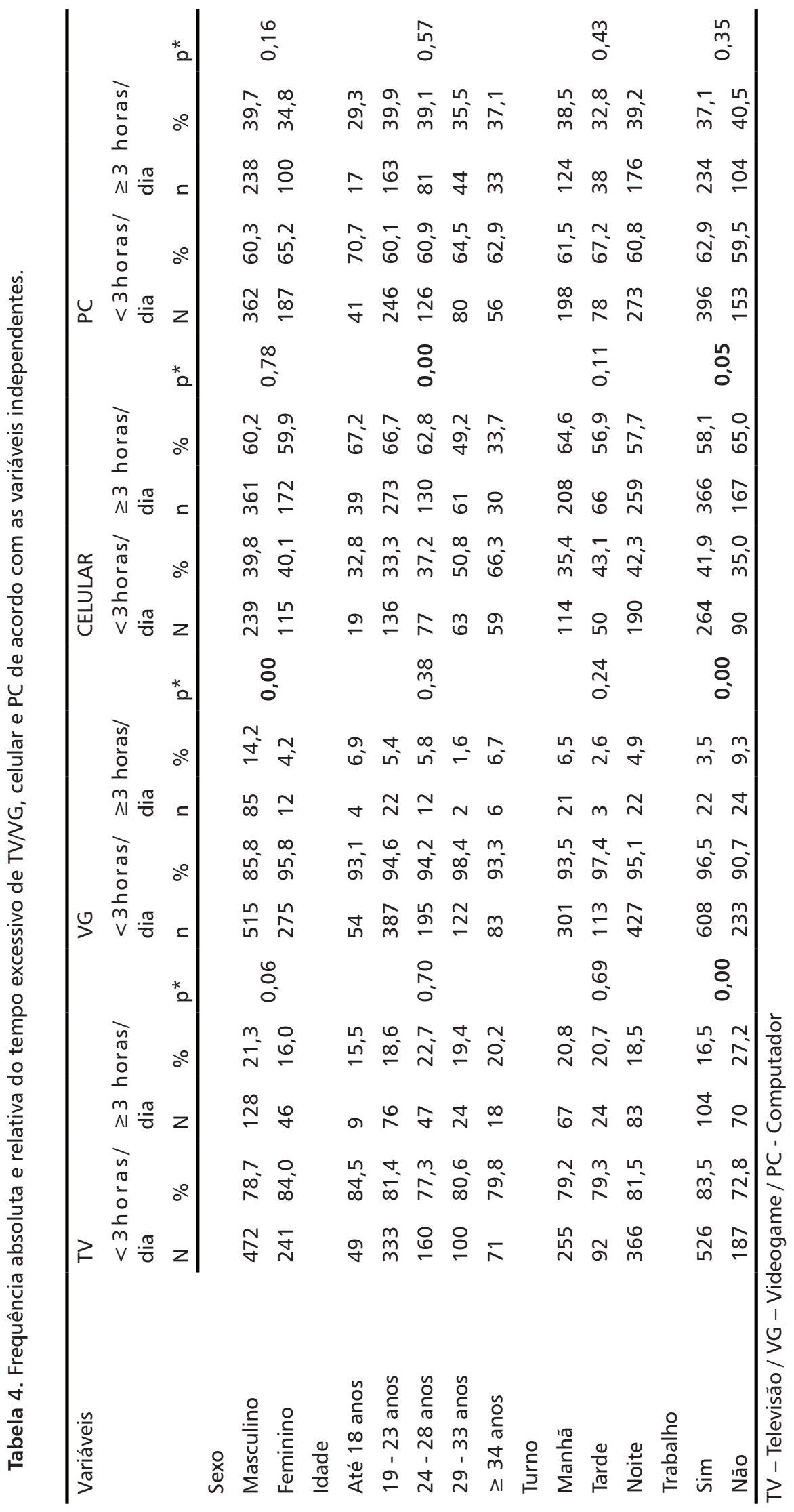


O CS esteve presente em $94,6 \%$ da população e está relacionado a atividades de baixa intensidade, com gasto energético reduzido, ou seja, o tempo em que os acadêmicos despendiam sentados na faculdade, em casa ou assistindo TV durante um dia típico da semana e do final de semana. Isso significa dizer que embora os indivíduos tenham, em sua maioria, sido classificados como ativos, também apresentaram CS, porque as variáveis não estiveram associadas, ou seja, uma é independente da outra.

Dito de outro modo, a prática de atividade física é um dos fatores que pode apresentar associação inversa com o tempo em atividades sedentárias. Todavia, alguns autores referem que atividades sedentárias independem da prática de atividade física, ou seja, o declínio em uma variável, não promove a elevação da outra (DIAS et al., 2014; MALIK; WILLET; HU, 2013; CRAEMER et al., 2012; AL-HAZZAA et al., 2011).

Esses resultados ratificam a importância de investimentos em pesquisas voltadas para a investigação do perfil dos profissionais da área da saúde e/ou ainda estratégias de intervenção para esta população, como ações educativas, visto que a prevalência de CS identificada nesse estudo foi semelhante à encontrada em estudos realizados em outros países (VEERMAN et al., 2012; SCHMID; RICCI; LEITZMANN, 2015; LEE et al., 2014).

As variáveis, sexo, turno e status ocupacional, exerceram efeito independente sobre o CS dos acadêmicos. Entretanto, o CS esteve diretamente associado ao TT, ou seja, o hábito de assistir TV, usar PC e aplicativos no celular, apresentou relação com o nível elevado de CS.

O tempo despendido em frente às telas tem sido considerado importante indicador de atividades sedentárias e está associado a inúmeros problemas de saúde, que incluem o CS, excesso de peso, entre outros. Diante disso, estratégias têm focado a diminuição do tempo diário gasto em frente à TV, VG, PC e celular, através de programas de intervenção, principalmente no combate ao tempo sedentário (FRIEDRI$\mathrm{CH}$ et al., 2013).

O TT total não foi diferente entre os sexos, todavia, os homens, passavam mais tempo em frente às telas quando comparado às mulheres. Além disto, o fato de não trabalhar foi responsável por elevar o TT em todos os indicadores. De maneira geral, a forma de entretenimento mais utilizada foi o celular, tanto entre os homens quanto entre as mulheres. Nesse estudo, muitos acadêmicos revelaram utilizar o celular (aplicativos em geral) por $1440 \mathrm{~min} /$ dia, o equivalente à 24 horas diárias. Todavia, esse dado foi superestimado, uma vez que não seria possível, pelo tempo dedicado ao sono.

A explicação para isto surge na "era smartphone". Uma recente pesquisa realizada com 500 universitários nos Estados Unidos relatou que em média os estudantes gastavam 278,67 minutos/dia (mais de quatro horas e meia) usando telefones celulares e outros 76,68 minutos/dia enviando mensagens de texto. Se a média chama atenção, o valor máximo relatado por muitos chega a $915 \mathrm{~min} / \mathrm{dia}$, o que significa dizer, 15,25 horas/dia (SENSÊVE, 2013).

Em caráter complementar, o uso das telas tem sido associado a outros fatores, como: nível de atividade física, hábitos alimentares e obesidade. Contudo, ainda que seja reconhecida a importância da prática de atividade física, os níveis de inatividade física são elevados na população, com implicações na saúde e no aumento do risco de doenças crônicas não transmissíveis (DCNT). Soma-se a isso a alta prevalência do CS (TAVARES et al., 2014).

A influência do TT na obesidade apresenta associação positiva, pois as mudanças no estilo de vida relacionadas ao CS justificam-se pelo fato de o tempo dedicado à prática de atividade física está sendo destinado ao uso dos aparelhos (TV, VG, PC e celular) Além disto, também está associado à ocorrência de hábitos alimentares menos saudáveis, visto que o telespectador preocupa-se mais com a programação que com o alimento ingerido nos intervalos de acesso (FLORES et al., 2012).

Desta maneira, os riscos relacionados à saúde para a era da geração digital são descritos em diversos estudos que comparam diversos indicadores de TT e diversos fatores associados. Por este motivo, é de suma importância verificar se há relação entre as variáveis. Em estatística, o procedimento que verifica a forma como as variáveis em estudo estão relacionadas é chamado de correlação.

Para evidenciar essa relação, pode-se recorrer ao coeficiente de correlação de Pearson ou ao coeficien- 
te de correlação de Spearman. Normalmente, o que orienta a escolha do tipo de coeficiente é o fato se os dados da pesquisa foram normais ou não. O coeficiente de correlação de Pearson é válido para dados normalmente distribuídos, e o de Spearman pode ser usado para dados que violam as suposições paramétricas, como bem orienta Field (2009).

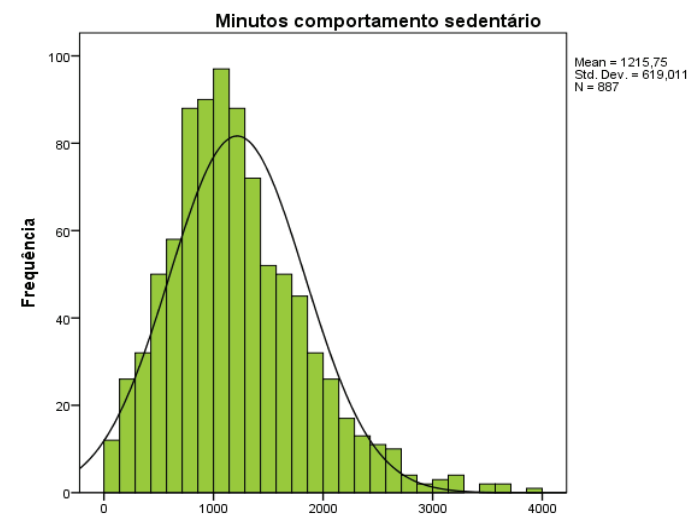

Ademais, solicitar no software os histogramas das variáveis sobrepostos por uma distribuição normal por meio da análise dos referidos histogramas, constata novamente se os dados fogem de uma distribuição normal (Figura 1 e 2).

Figura 1. Histograma Tempo do Comportamento Sedentário em Minutos e em Horas.

Fonte: A pesquisadora (2015)
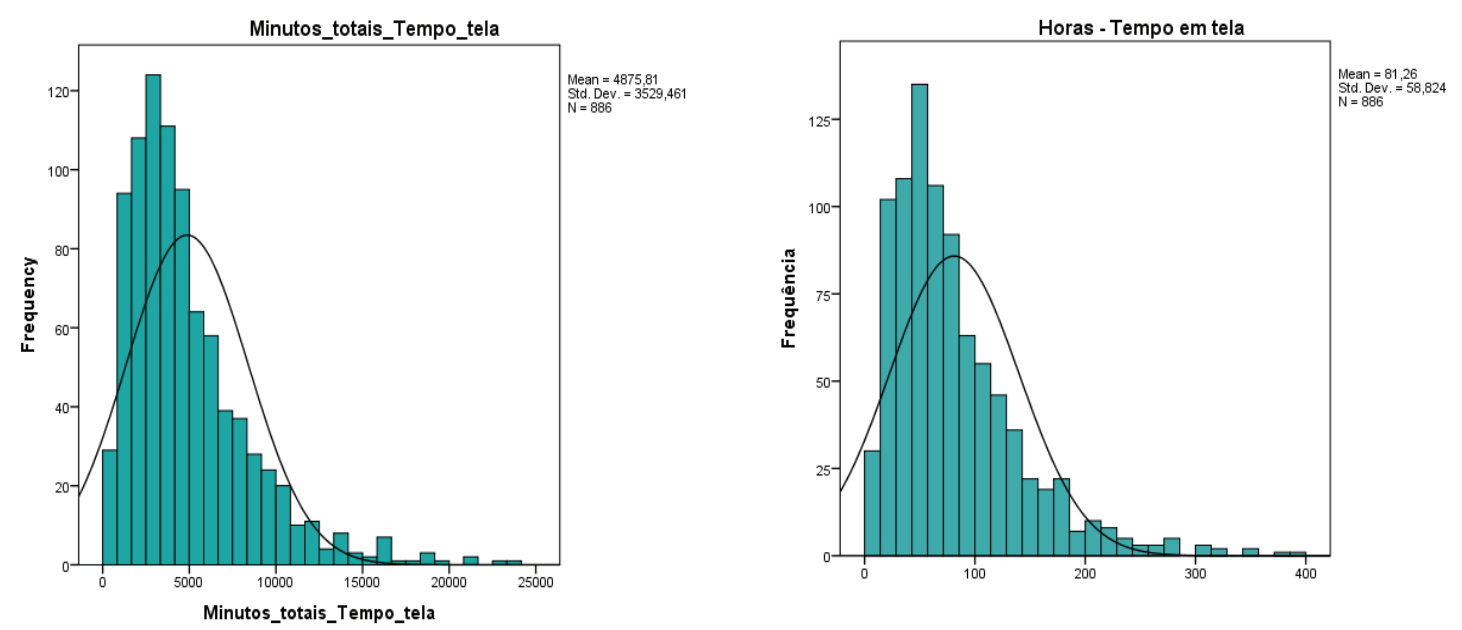

Figura 2. Histograma do Tempo em tela em Minutos e em Horas.

Fonte: A pesquisadora (2015)

Isso posto, na análise do relacionamento existente entre o comportamento sedentário e o tempo em tela (televisão, videogame, computador e aplicativos do celular) utiliza-se o teste de Spearman, eviden-

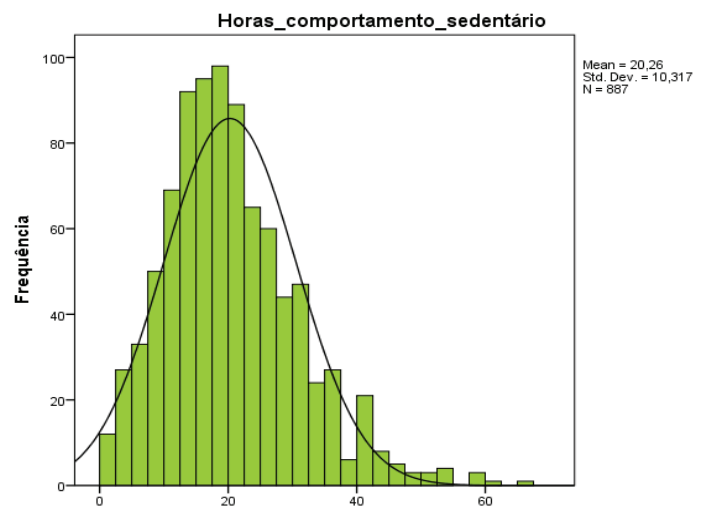


Quadro 02. Correlação de Spearman'srho

- Comportamento sedentário - Tempo em tela.

\begin{tabular}{|l|l|l|}
\hline & & $\begin{array}{l}\text { Tempo em } \\
\text { tela (Min) }\end{array}$ \\
\hline \multirow{2}{*}{$\begin{array}{l}\text { Comportamento } \\
\text { sedentário (Min.) }\end{array}$} & (Spearman'srho) & $0,352^{* *}$ \\
\cline { 2 - 3 } & $\mathrm{N}$ & 0,000 \\
\cline { 2 - 3 } & $\mathrm{N}$. & 886 \\
\hline
\end{tabular}

**Correlação é significativa (Sig. $<0,05$ ).

Fonte: A pesquisadora (2015).

A interpretação dos resultados desse teste segue alguns parâmetros, em primeiro lugar se entende que:

Um coeficiente de +1 indica que as duas variáveis são perfeitamente correlacionadas de forma positiva, assim, enquanto uma variável aumenta, a outra aumenta proporcionalmente. Inversamente, um coeficiente de - 1 indica um relacionamento negativo perfeito: se uma variável aumenta, a outra diminui por um valor proporcional. Um coeficiente 0 indica ausência de relacionamento linear, isto é, se uma variável muda, a outra permanece praticamente igual (FIELD, 2009, p. 129).

Em segundo lugar, um coeficiente de correlação: igual a +0,1 indica uma correlação pequena; igual a $+0,3$ indica uma correlação média; e, igual $+0,5$ indica uma correlação grande (FIELD, 2009). Nesse sentido, podemos dizer que a correlação existente entre o CS e o TT foi média ( $\square$ de Spearman $=0,352$ ).

Ademais, as limitações desse estudo incluem um número pequeno de pesquisas com acadêmicos de Educação Física e o método utilizado para determinar o CS não oportuniza medidas precisas, tais como o acelerômetro. Isto porque o IPAQ assim como outros questionários, possui potencial para vieses que superestimam a prática de atividade física e subestimam o CS. Além disto, estudos transversais possuem limitações metodológicas, pois não avaliam causa e efeito. Contudo, os resultados descritos nesse estudo foram similares aos que têm sido descritos em outros estudos transversais.

Estes aspectos são relevantes e devem ser considerados em programas de políticas públicas voltados à saúde e estratégias para reduzir o TT e combate ao tempo sedentário em universitários.

\section{CONCLUSÃO}

De acordo com os achados no presente estudo, conclui-se que o comportamento sedentário está diretamente associado ao tempo de tela. O estudo avaliou uma quantidade expressiva de indicadores de atividade física e CS, oferecendo contribuição pioneira sobre o CS nos acadêmicos de Educação Física da capital de Fortaleza e Região Metropolitana do Ceará.

Nesse sentido, os resultados encontrados podem ser utilizados na projeção de intervenções no âmbito educacional com orientações para a diminuição do CS e aumento da prática de atividade física. Por conseguinte, estudos que associem diversos fatores e indicadores de TT tornam-se importantes para direcionamentos futuros de políticas públicas voltadas à saúde e combate ao CS, além de estratégias para reduzir o TT entre os acadêmicos.

A realização de estudos similares em outras localidades permitirá o conhecimento sobre os níveis encontrados para populações congêneres, permitindo um delineamento mais fidedigno dos fatores que induzem ao CS.

\section{REFERÊNCIAS}

AL-HAZZAA, H.M.; ABAHUSSAIN, N.A.; AL-SOBAYEL, H.I.; QAHWAJ, D.M.; MUSAIGER, A.O. Physical activity, sedentary behaviors and dietary habits among Saudi adolescents relative to age, gender and region. Int J Behav Nutr Phys Act. 2011.

ARMSTRONG, J.;DOROSTY,A. R.;REILLY, J. J. Child Health Information Team, Emmett PM. Coexistence of social inequalities in under nutrition and obesity inpreschool children: population based cross sectional study. Archivesof Disease in Childhood, v. 88, n.1: p. 671-675, 2003.

CIESLACK, F.; CAVAZZA, J. F.; LAZAROTTO, L.; TITSKI, A. C. K.; STEFANELLO, J. M. F.; LEITE, N. Análise da qualidade de vida e do nível de atividade física em universitários. Revista Educação Física, v.23, n.2: p. 251-260, 2012.

CRAEMER, M.; DECKER, E.;BOURDEAUDHUIJI, I.; VEREECKEN, C.; DEFORCHE, B.; MANIOS, Y., et al. Correlates of energy balance-related behaviours in preschool children: asys tematic review. Obesity Revist, v.13, n.1: p. 13-28, 2012.

DIAS, P. J. P.; DOMINGOS, I. P.; FERREIRA, M. G.; MURARO, A. P.; SICHIERI, R.; SILVA, R. M. V. Prevalência e fatores as- 
sociados aos comportamentos sedentários em adolescentes. Revista Saúde Pública, São Paulo, v.48, n.2, Abr. 2014.

FIELD, A. Descobrindo a estatística usando o SPSS. 2. ed. Porto Alegre, RS: Artmed, 2009.

FLORES, P. T; LIMA, K.; PUCCI, V.; SOUZA, J. G; NORNBERG, M. L.; BENEDETTI, F. J. Influência do tempo em frente à televisão na obesidade e no consumo alimentar em crianças e adolescentes. Revista de nutrição, Rio Grande do Sul, 2012.

FONTES, A. C. D.; VIANNA, R. P. T. Prevalência e fatores associados ao baixo nível de atividade física entre estudantes universitários de uma universidade pública da Região Nordeste - Brasil. Revista Brasileira de Epidemiologia, São Paulo, v.12, n.1, Mar. 2009.

FRIEDRICH, R. R.; POLET, J. P.; SCHUCH, I.; WAGNER, M. B. Effect of intervention programs in schools to reduce screen time: a meta-analysis. Jornal de Pediatria, Rio de Janeiro, v.90, p. 232-241, 2014.

IPAQ - International Physical Activity Questionnaire. Guidelines for data processing and analysis of the international physical activity questionnaire (IPAQ). 2005.

JUNIOR, E. F. C.; BARRETO, L. A.; OLIVEIRA, J. A. A.; ALMEIDA, P. C.; LEITE, J. A. D. Avaliação do nível de atividade física e fatores associados em estudantes de medicina de Fortaleza-CE. Revista Brasileira Ciência e Esporte, Florianópolis, v.34, n.4: p. 955-967. Out/Dez 2012.

LEE, S. T.; WONG, J. E.; SHANITA, S. N.; ISMAIL, M. N.; DEU RENBERG, P.; POH, B. Daily Physical Activity and Screen Time, but Not Other Sedentary Activities, Are Associated with Measures of Obesity during Childhood. Int. J. Environ. Res. Public Health. Dez. 2014

MALIK, V. S.; WILLETT, W. C.; HU, F. B. Global obesity: trends, risk factors and policy implications. Nat Rev Endocrinol, v.9, n.1: p. 13-27, 2013.

MEDEIROS, J.; VIEIRA, F. G. D.; NOGAMI, V. K. C. O consumo de tablets entre estudantes universitários. Revista de Administração da UNIMEP. v.11, n.2, 2013
OLIVEIRA, T. C.; SILVA, A. A. M.; SANTOS, C. J. N.; SILVA, J. S.; CONCEIÇÃO, S. I. O. Atividade Física e Sedentarismo em escolares da rede pública e privada de ensino em São Luís. Rev. Saúde Pública, São Paulo, v.44, n.6, p 996-1004, 2010.

SCHMID, D.; RICCI, C.; LEITZMANN, M. F. Associations of Objectively Assessed PhysicalActivity and Sedentary Time with All-CauseMortality in US Adults: The NHANES Study. Journal Plos One, United States, v.10, n.3, Mar. 2015.

SENSÊVE, B. Uso exagerado de smartphone. Disponível em <http://sites.uai.com.br/app/noticia/saudeplena/noticias/2013/12/30/noticia_saudeplena,146979/uso-exagerado-de-smartphones-interfere-no-desempenho-escolar-diz-estu.shtml>. Acesso em: 23 de nov. 2015.

SILVA, G. S. F.; BERGAMASCHINE, R.; ROSA, M.; MELO, C.; MIRANDA, R.; FILHO, M. B. Avaliação do nível de atividade física de estudantes de graduação das áreas saúde/biológica. Revista Brasileira de Medicina do Esporte, v.13, n.1, Jan/Fev 2007.

STAINO, V. S.; PEREIRA, J. O.; RODRIGUES, H. W. G.; ANDRADE, A. G. P.; JÚNIOR, M. B. Atividade Física e comportamento sedentário em universitários do curso de Educação Física em Belo Horizonte. Coleção Pesquisa em Educação Física, Belo Horizonte, v.9, n.6, 2010.

TAVARES, L. F.; CASTRO, I. R. R.; CARDOSO, L. O.; LEVY, R. B.; CLARO, R. M.; OLIVEIRA, A. F. Validade de indicadores de atividade física e comportamento sedentário da Pesquisa Nacional de Saúde do Escolar entre adolescentes do Rio de Janeiro, Brasil. Caderno de Saúde Pública, Rio de Janeiro, v.30, n.9, p. 1861-1874, set. 2014

VASCONCELLOS, M.B, ANJOS, L.A, VASCONCELLOS, M.T.L. Estado nutricional e tempo de tela de escolares da Rede Pública de Ensino Fundamental de Niterói, Rio de Janeiro, Brasil. Cad. Saúde Pública, Rio de Janeiro, v. 29, n. 4: p. 713-722, Abr. 2013.

VEERMAN, J. L.; HEALY, G. N.; COBIAC, L. J.; VOS, T.; WINKLER, L. A. H.; OWEN, N.; DUNSTAN, D. Television viewing time and reduced life expectancy:a life table analysis. $\mathrm{Br} J$ Sports Med, v. 46, p.927-930, 2012.

World Health Organization WHO. Global status report on non communicable diseases. Geneva: 2010 